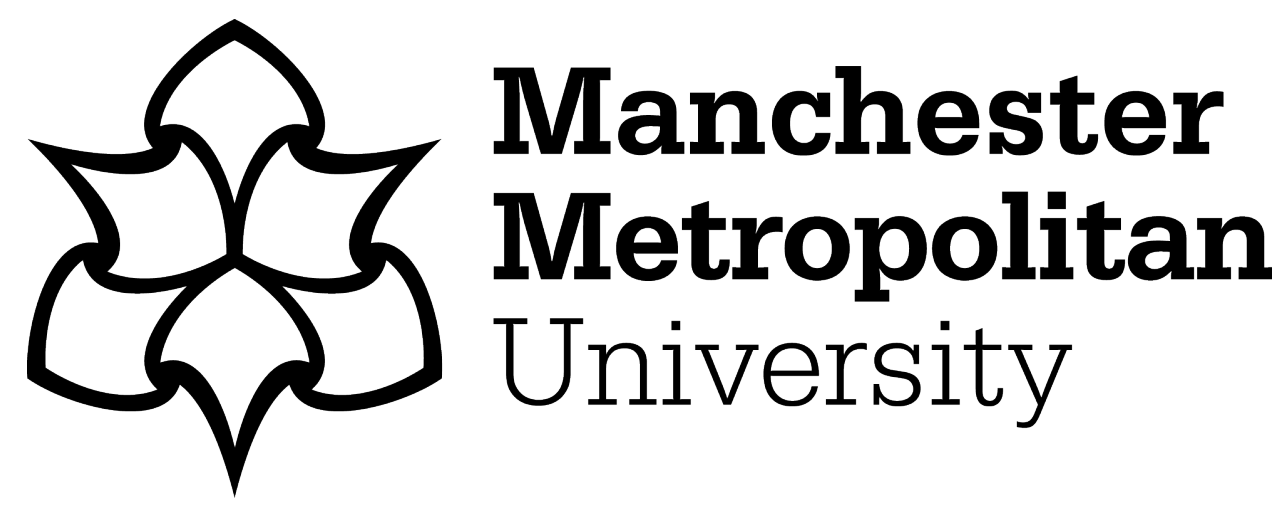

Di Giulio, I, McFadyen, BJ, Blanchet, S, Reeves, ND ORCID logoORCID: https://orcid.org/0000-0001-9213-4580, Baltzopoulos, V and Maganaris, CN (2019) Mobile phone use impairs stair gait: A pilot study on young adults. Applied Ergonomics, 84. ISSN 0003-6870

Downloaded from: https://e-space.mmu.ac.uk/625142/

Version: Accepted Version

Publisher: Elsevier

DOI: https://doi.org/10.1016/j.apergo.2019.103009

Please cite the published version 


\title{
Mobile phone use impairs stair gait: A pilot study on young adults
}

\author{
Irene Di Giulio $^{\text {a, }}$, Bradford J. McFadyen ${ }^{\text {b }}$, Sophie Blanchet ${ }^{\text {b,c }}$, Neil D. Reeves ${ }^{\mathrm{d}}$, \\ Vasilios Baltzopoulos $^{e}$, Constantinos N. Maganaris ${ }^{e}$ \\ ${ }^{\text {a }}$ Centre for Human \& Applied Physiological Sciences, King's College London, London, UK \\ ${ }^{\mathrm{b}}$ Centre for Interdisciplinary Research in Rehabilitation and Social Interaction/Universite Laval, Quebec City, Canada, G1M 2S8 \\ ${ }^{\mathrm{c}}$ Memory and Cognition Laboratory, INSERM UMR 894, Centre of Psychiatry and Neuroscience and Institute of Psychology, Universite Paris Descartes, Sorbonne Paris \\ Cite, Paris, France \\ ${ }^{\mathrm{d}}$ Research Centre for Musculoskeletal Science \& Sports Medicine, Manchester Metropolitan University, Manchester, M1 5GD, UK \\ ${ }^{\mathrm{e}}$ Research Institute for Sport and Exercise Sciences, Liverpool John Moores University, Liverpool, UK
}

\section{A R T I C L E I N F O}

\section{Keywords:}

Dual task

Locomotor performance

Control cost

Information processing interference

\begin{abstract}
A B S T R A C T
Human movement control requires attention to accurately tune motor commands in response to environmental changes. Dual task paradigms are used to test the role of attention on motor performance. Usually the tasks used have little resemblance with every day experience. Here we ask: Does a common cognitive task, such as a mobile phone conversation, compromise motor performance on stairs?

Eight young participants negotiated an instrumented seven-step staircase. Stair negotiation while talking on a mobile phone was compared to normal stair negotiation. Stepping parameters, jerk cost (measure of smoothness of locomotion) and step clearance were measured.

When talking on a mobile phone, participants' overall body velocity (mean(sd): Ascent $0.534(0.026)$ vs 0.511 $(0.024) \mathrm{m} / \mathrm{s}$, Descent $0.642(0.026)$ vs $0.511(0.024) \mathrm{m} / \mathrm{s}$, No phone/Phone respectively) and cadence decreased significantly (Ascent 75.8(5.8) vs 65.6(4.4) steps/min, Descent 117.4(4.2) vs 108.6(6.0) steps/min, No Phone/ Phone respectively). Pelvis and feet jerk cost also changed significantly, mostly decreasing with phone use. Foot clearance did not show significant changes between No Phone and Phone conditions.

These pilot results show that, even for young, healthy and cognitively intact individuals, talking on a mobile phone whilst negotiating a staircase induces measurable changes in motor performance. Participants moved slowly but more smoothly, reducing the motor control cost, possibly at the expense of movement accuracy. The reduction in motor performance is likely to be due to the difficulty in integrating the two sub-tasks. These results suggest that even young, healthy individuals show stair gait impairment when simultaneously negotiating stairs and performing another cognitive task, such as talking on the phone.
\end{abstract}

\section{Introduction}

Human motor tasks such as standing, walking (Lajoie et al., 1993; Woollacott and Shumway-Cook, 2002) and stair negotiation (Madehkhaksar and Egges, 2016) require attention. Individuals often multitask, for example they walk while having a conversation in person or on the phone. Multitasking involves divided attention and consequently performance is affected, indicating a strong link between cognitive load and motor control (Madehkhaksar and Egges, 2016; Verghese et al., 2007; Nasar et al., 2008; Lamberg and Muratori, 2012; Plummer et al., 2015).

Multitasking can be investigated using dual-task tests. Traditionally, two main paradigms have been used: real-life observations and laboratory-based experiments. Real-life observations are not artificial, but the quantities that can be measured are limited (e.g. increased time spent crossing the road whilst talking on a mobile phone) (Hatfield and Murphy, 2007). Laboratory-based studies accurately quantify standing or walking, but the cognitive tasks used are not necessarily real, such as arithmetic and spelling tasks, spatial and non-spatial memory tasks or memorising word lists (Pellecchia, 2003). The present study exploited the benefits of both paradigms and a new protocol was designed to investigate two real-life tasks, talking on the phone and stair negotiation, in the laboratory. This approach is promising because quantification is needed in this research field. However, here we used phone conversation rather than texting or typing (Demura and Uchiyama,

\footnotetext{
* Corresponding author.

E-mail address: irene.di giulio@kcl.ac.uk (I. Di Giulio).
} 
2009; Licence et al., 2015; Schabrun et al., 2014) because we aimed to measure the effect of the conversation without involving the visuo-motor interference as in texting (Timmis et al., 2017; Ioannidou et al., 2017).

Unspecified telephone use is related to $\sim 11,000$ home and leisure accidents in the UK per year (HASS and LASS, 2002). This report is more than a decade old, and it is highly likely that the increase in phone use may be related to a higher number of accidents. Talking on a mobile phone has been highly studied in conjunction with driving (Strayer and Johnston, 2001; Patten et al., 2004), walking (Nasar et al., 2008; Lamberg and Muratori, 2012; Plummer et al., 2015) or crossing the road (Neider et al., 2010, 2011; Stavrinos et al., 2011; Schwebel et al., 2012). Performance was negatively affected by mobile phone use, as measured by increased time to complete the task and risky behaviours. No difference was found between hand-held and hand-free mobile phones (Nasar et al., 2008; Strayer and Johnston, 2001). This suggests that diversion of attention and the conversation itself interfere with encoding of information in working memory (Neider et al., 2010), where new and stored information are processed during reasoning, comprehension and memory updating.

Stair negotiation was chosen as the simultaneous motor task here. Compared to level walking, stair negotiation poses a higher musculoskeletal load to move the body mass forwards and up/downwards with concentric and eccentric muscle contractions, and challenges balance control and coordination of muscles in the single support phase (in particular during descent) (Madehkhaksar and Egges, 2016; Riener et al., 2002; Reeves et al., 2008, 2009). Stair negotiation is often investigated because falls are common when negotiating a staircase, with about 300,000 accidents per year on the stairs in the UK alone (HASS and LASS, 2002). Stair negotiation is a suitable motor task because it requires attention and planning and potentially error correction; working memory and information processing are engaged to respond to changes in the environment.

The two tasks chosen here (phone conversation during stair negotiation) require attention, do not increase difficulty indiscriminately and thus avoid floor or ceiling effects (Schaefer and Schumacher, 2010), and are fairly common, which excludes learning and habituation components that could affect the results. In the present pilot study, a small group of young participants were tested using this novel protocol to measure if and how talking on a mobile phone affects stair locomotion.

We hypothesise that stair negotiations will show changes when talking on the phone consistent to a compensatory method to reduce fall risk, even in a population of young adults. Such changes, reflecting the increased difficulty of an ecological cognitive task on movement control for a healthy young population, would be of greater concern to populations with decreased cognitive and physical capacity.

\section{Methods}

\subsection{Ethical approval}

Participants gave written informed consent to this experiment which conformed to the standards set by the Declaration of Helsinki and was approved by the ethics committee of the Faculty of Science and Engineering, Manchester Metropolitan University.

\subsection{Participants and procedure}

Eight healthy young participants with no history of brain injury or other neurological conditions were tested (six males, two females; mean (standard deviation 'SD'); age, 27(4) years; height, 1.75(0.12) m; mass, $74.2(18.2) \mathrm{kg}$, self-reported leg and hand dominance 1 left, 6 right and 1 ambidextrous). Participants were asked to ascend and descend (stepover-step, i.e. one foot only on each stair step) a seven-step staircase at their self-selected pace and most comfortable manner (Fig. 1). They performed the task barefooted to control for the potential influence of footwear on performance and walking speed (Simoneau et al., 1991; Menz et al., 2003). Stair negotiation was preceded and followed by walking on a flat $2 \mathrm{~m}$-walkway. The stair steps were within standard guidelines, $275 \mathrm{~mm}$ deep and $175 \mathrm{~mm}$ high (Government UK, 2013), therefore no practice trial was performed. Handrails were provided on either side of the staircase and participants were told that they could use them, if they wanted to do so, but they were never used.

Two conditions (three trials each) were compared: normal stair negotiation (No Phone) and stair negotiation while talking on a mobile phone (Phone). As part of a larger study, participants always negotiated the staircase whilst talking on the phone first.

In the Phone trials, participants were engaged in a conversation with an operator who asked loosely scripted questions that engaged working memory (Table 1). The operator chose between different streams of conversation and would follow-up on any of them to make sure that the conversation would flow for the entire duration of the experiment. Participants were asked to talk with the operator (in another room) as if they were talking with an acquaintance about relatively trivial matters. Participants were not given specific instructions on how to perform the trials, but were asked to walk on the stairs and continue the conversation on the phone until told to stop at the end of the set of trials. Because each trial lasted 10s and 6 trials per participant were collected, the conversation lasted about $2 \mathrm{~min}$. Participants received a visual go signal at the beginning of each trial.

\subsection{Apparatus and measurement}

The seven-step staircase had four $300 \quad 500 \mathrm{~mm}$ force platforms (model 9260AA3, Kistler Instrumente, CH-8408 Winterthur, Switzerland) embedded in steps 2-5 (considering step one as the bottom step or first raised step, see Fig. 1). The force platforms were used to assess when the foot landed and lifted-off the step.

The staircase was situated in a volume covered by a ten-camera optoelectronic movement analysis system (Vicon Motion Systems, Oxford, UK). Retro-reflective markers were attached to the participant's skin or tight fitting clothes using the Plug-In-Gait model, with additional markers on left and right dorsal aspect of the second toe distal tip, fifth metatarsal head, medial malleolus, lateral calcaneus and medial calcaneus. Data were collected at $100 \mathrm{~Hz}$. Marker trajectories were filtered

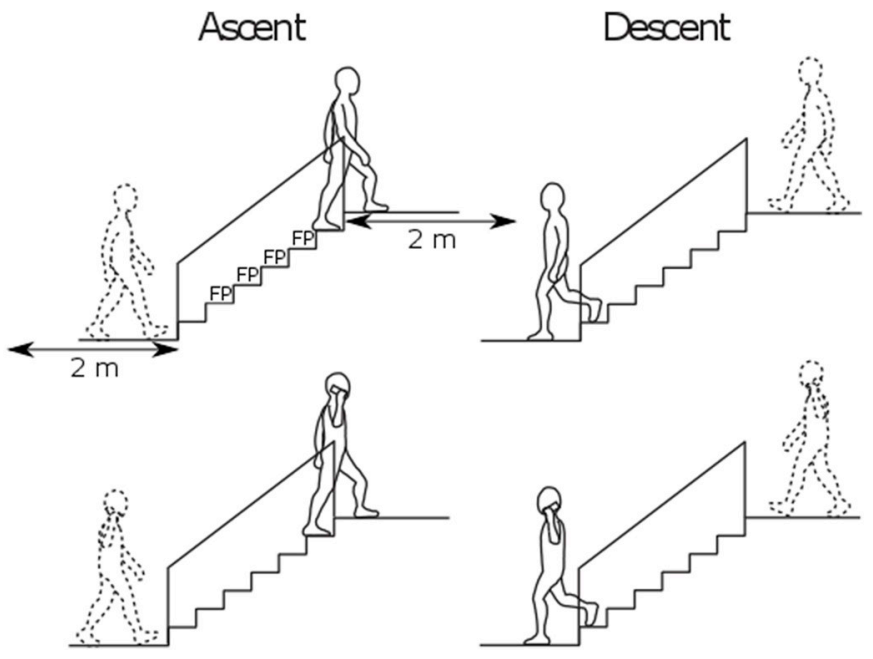

Fig. 1. Apparatus.

The seven-step instrumented staircase. Step size: height $175 \mathrm{~mm}$, going 275 $\mathrm{mm}$. Force platforms (FP) were embedded in step 2-5. Handrails were provided on each side. A $2 \mathrm{~m}$ walkway was provided before and after the stair. Stair ascent and descent were measured. Two conditions were compared: normal stair negotiation (No Phone) and stair negotiation while talking on a mobile phone (Phone). 
Table 1

Questions for conversation. Set of questions given to the operator sitting in a room beside the laboratory to be used when phoning the participant to start a conversation. The questions were divided into topics. The operator followed the questions, but was free to pursue some of them if the conversation became more engaging for the participant on one particular topic. Adapted from Neider and colleagues (Neider et al., 2011).

\begin{tabular}{ll}
\hline Topic & Question \\
\hline Start & I am ..., do you have 5 min to talk? \\
Holidays & Good, so how are you? \\
& Do you have any plans for the weekend? \\
& Have you booked your holidays yet? \\
Movie & Where will you go? \\
& Have you seen any movies recently? \\
Music & Who was in it? \\
& What is your favourite type of music? \\
Books & What is your favourite band? \\
& Have you read any books recently? \\
Hometown & What are your favourite books? \\
& What was the book about? \\
Close & Where is your home town? \\
\hline
\end{tabular}

using a fourth order, zero-lag, low-pass Butterworth filter with $20 \mathrm{~Hz}$ cut-off.

Handrails were provided on both sides of the staircase. A harness system suspended from a trolley and girder on the ceiling of the laboratory was available to ensure safety.

\subsection{Kinematic quantities}

All the quantities were calculated with custom scripts in Matlab (Mathworks, Natick, US). Foot events at each step were calculated. On the steps with force plates, the foot landing and lift-off were calculated using the ground reaction force (above and then below 10N threshold respectively). For the other steps, a method derived by Pijnappels and colleagues (Pijnappels et al., 2001) was used. In ascent, landing was defined as the local maxima of the front foot velocity trace (from 2 nd toe tip, 2nd and 5th metatarsal head), and lift-off as the local maxima of the front foot acceleration trace. In descent, landing was defined as the local maxima of the front foot acceleration trace and lift-off as the local maxima of the front foot velocity trace. The differences between events calculated with the force plates and from kinematic data was $2 \mathrm{~ms}$.

For the quantities calculated, averages over both steady state ('SS', between step 2 and 5) and the whole staircase ('O', between step 1 and 7) were calculated. Overall data showed behaviours related to the whole staircase including the transitional phases. These may hide the differences more purely related to stair negotiation, which are captured in the steady state version of the quantities. Hence the two types of quantities were provided when a difference in behaviour due to the transition between level and stair gait was relevant.

Body velocityss,o $(\mathrm{m} / \mathrm{s})$. The mean position of the upper body segment (proxy for whole body) in the direction of travel was calculated from markers on the head (front and back, left and right), C7 (seventh cervical vertebra), T10 (tenth thoracic vertebra), sternum, clavicle, and pelvis (left and right anterior and posterior iliac crests LASI, RASI, LPSI, RPSI). Velocity and acceleration were calculated using a band-pass Remez differentiator filter (IEEE, 1979) with a band $1 \mathrm{~Hz}$ to 0.9 $100 \mathrm{~Hz}$. This method minimises error between desired and actual frequency response.

Cadence $_{\text {SS,o }}$ (steps/min) calculated as the foot strike at each step in seconds, converted into steps $/ \mathrm{min}$.

Step timing variabilityss,o (s) calculated as the standard deviation of the time between successive foot strikes (dispersion of data relative to the mean).

Acceleration root mean square $e_{0}$ (RMS). The root mean square of the upper body acceleration was calculated to give an indication of the dispersion of the data relative to 0 (Menz et al., 2003).

Jerk cost $\mathrm{SS}, \mathrm{O}\left(\mathrm{m}^{2} / \mathrm{s}^{5}\right)$ in the direction of travel (Nelson, 1983; Schneider and Zernicke, 1989)

Jerk cost $\frac{\int j e r k^{2}}{2}$

where jerk is the derivative of the acceleration in the direction of travel over time (successive zero-lag derivatives and filtering), and the integration interval is the time of the movement. Smoother locomotion is consistent with lower jerk cost (Hreljac, 1993). The jerk cost was calculated for upper body, pelvis and feet. The upper body was used for consistency with the quantities calculated previously (e.g. body velocity and using the same markers). The jerk of the pelvis (from LASI, RASI, LPSI, RPSI) was calculated because it has been identified as the body segment that represents whole body smoothness of locomotion (Menz et al., 2003). The foot jerk cost (from 2nd and 5th metatarsal head, 2nd toe tip) was calculated because the maximum smoothness principle assumes that the objective of motor coordination should be expressed in the coordinate system in which the movement planning is assumed to occur and, in walking, this is identified as the feet (Hreljac, 1993). This quantity was calculated for dominant and non-dominant foot.

Step clearancesss $(\mathrm{m})$ calculated as the minimum distance between the front (ascent: markers on 2nd toe tip, 2nd and 5th metatarsal head) and rear foot (descent: heel, medial and lateral side of the calcaneus) and each step edge in the direction of travel (horizontal plane) and in the vertical plane.

\subsection{Statistical analysis}

Ascent and descent were analysed separately using SPSS (IBM SPSS ver.24). A paired, two tailed Wilcoxon signed rank test was used to assess the difference between motor performance with and without talking on a mobile phone for every variable. Conversation performance was not assessed. For each participant, averages of up to three trials for each condition were used (three trials with phone and three without phone). For participant 1, two trials with mobile phone had to be excluded for technical difficulties (one for ascent, one for descent). For participant 4 , one stair descent trial with mobile phone had to be excluded. To account for multiple comparisons, p-values were adjusted using the False Discovery Rate (FDR) method with q 0.05 and $\mathrm{c}(\mathrm{V})$ 1.

The effect size was calculated with the standardised difference score described using the formula below.

$d \frac{\text { mean } Q_{\text {Phone }} Q_{\text {No Phone }}}{\text { standard deviation } Q_{\text {Phone }} \quad Q_{\text {No Phone }}}$

where $\mathrm{Q}$ indicates the quantity considered.

To investigate whether our results indirectly reflected a difference in body velocity, a Pearson's correlation was run between all the quantities and body velocity.

Results are reported in the figures as mean standard error ( s.e. m.). Significance level was 0.05 .

\section{Results}

All participants freely held the mobile phone in their dominant hand. The ambidextrous participant held the mobile phone in her left hand. Participants never used the handrails.

\subsection{Ascent}

When talking on the phone, the body velocity was slower (overall and steady state $\mathrm{p}_{\text {adj_o }} \quad 0.006$, effect size $\mathrm{d}_{\mathrm{O}} \quad 1.64$; $\mathrm{p}_{\text {adj_ss }} \quad 0.006, \mathrm{~d}_{\mathrm{SS}}$ 1.71; Fig. 2), cadence was, on average, about $10 \mathrm{steps} / \mathrm{min}$ lower at 
steady state ( $\mathrm{p}_{\text {Adj_O }} \quad 0.067, \mathrm{~d}_{\mathrm{O}} \quad 0.64$; $\mathrm{p}_{\text {Adj_Ss }} \quad 0.006, \mathrm{~d}_{\mathrm{SS}} \quad 1.19$; Fig. 2), and step timing variability was higher at steady state (padj_O $0.313, \mathrm{~d}_{\mathrm{O}} \quad 0.29$; $\mathrm{p}_{\text {Adj_SS }} \quad 0.023, \mathrm{~d}_{\mathrm{SS}} \quad 1.05$; Fig. 2). No difference was found for acceleration RMS ( $\mathrm{p}_{\mathrm{O}} \quad 0.675, \mathrm{~d}_{\mathrm{O}} \quad 0.32$ ).

The jerk cost in the direction of travel was not different between the two conditions for the upper body ( $\mathrm{p}_{\text {Adj-O }} \quad 0.167, \mathrm{~d}_{\mathrm{O}} \quad 0.43$; $\mathrm{p}_{\text {Adj_SS }}$ $0.201, \mathrm{~d}_{\mathrm{SS}} \quad 0.06$; Fig. 3 ), for the pelvis segment (padj_O $\quad 0.325, \mathrm{~d}_{\mathrm{O}}$

0.28 ; $\mathrm{p}_{\text {Adj-SS }} \quad 0.162, \mathrm{~d}_{\mathrm{SS}} \quad 0.27$; Fig. 3 ), and was lower when talking on the phone for the non-dominant foot overall (p $\mathrm{p}_{\text {Adj-O }} \quad 0.019, \mathrm{~d}_{\mathrm{O}}$ 1.26; p pdj_SS $\quad 0.674, \mathrm{~d}_{\mathrm{SS}} \quad 0.35$; Fig. 3 ).

No significant differences were shown for mean steady state clearances in the lead or trail foot in the vertical or horizontal direction ( $\mathrm{p}>$ 0.05; Fig. 4). The effect size was moderate for all clearances except for the horizontal trail foot $\left(\mathrm{d}_{\text {Vertical_Lead }} \quad 0.42\right.$; $\mathrm{d}_{\text {Vertical_Trail }} \quad 0.41 ; \mathrm{d}_{\text {Hor- }}$ izontal_Lead 0.28 ; $d_{\text {Horizontal_Trail }} 0.09$ ).

Body velocity highly correlated with cadence $\left(\mathrm{r}_{\mathrm{O}} \quad 0.55\right.$, $\mathrm{p}_{\mathrm{O}} \quad 0.028$; $\left.\mathrm{r}_{\mathrm{SS}} \quad 0.89, \mathrm{p}_{\mathrm{SS}}<0.001\right)$ and non-dominant foot jerk cost $\left(\mathrm{r}_{\mathrm{O}} \quad 0.86, \mathrm{p}_{\mathrm{O}}<\right.$ $0.001 ; \mathrm{r}_{\mathrm{SS}} \quad 0.51, \mathrm{p}_{\mathrm{SS}} \quad 0.043$ ), but not with step timing variability ( $\mathrm{p}_{\mathrm{O}}$

0.43 , pss 0.32), acceleration RMS ( $p_{s s} \quad 0.93$ ), pelvis jerk cost ( $p_{0}$

0.20 , pss 0.19 ), dominant foot jerk cost ( $p_{0} \quad 0.052$, pss 0.13 ), or clearances ( $\mathrm{p}_{\text {Vertical_Lead }} \quad 0.77$; p pertical_Trail 0.33 ; P Porizontal_Lead 0.20; PHorizontal_Trail 0.18 ).

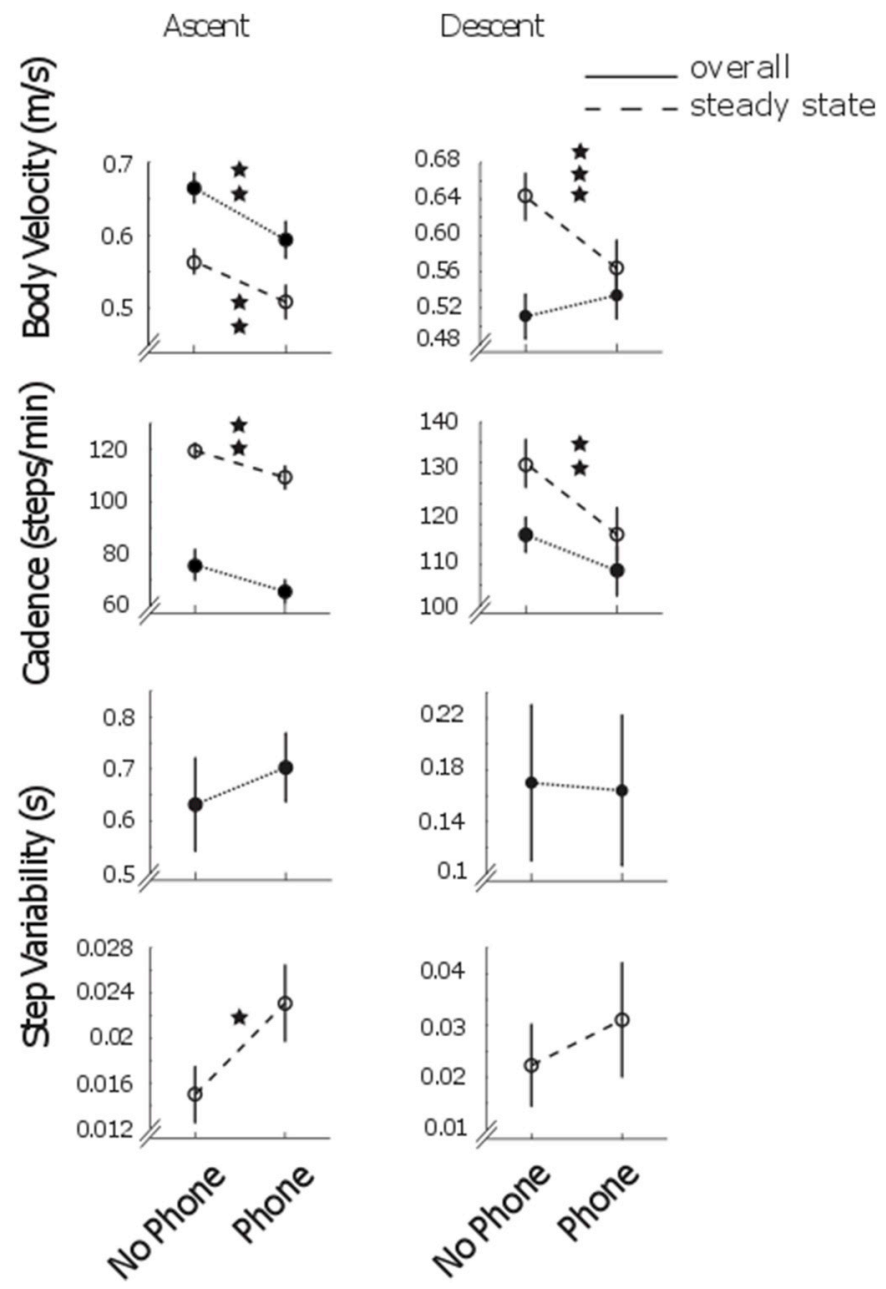

Fig. 2. Kinematic variables.

Group mean and standard error of the mean (s.e.m.) body velocity in the direction of travel, cadence and step variability. Overall (closed symbols, dotted lines) and steady state (open symbols, dashed) quantities reported for stair negotiation only (No Phone) and whilst talking on a mobile phone (Phone), for stair ascent (left) and descent (right). One star represents $\mathrm{p}<0.05$, two stars $\mathrm{p}$ $<0.01$, three stars $\mathrm{p}<0.001$.

\subsection{Descent}

When talking on the phone, body velocity was reduced in the steady state part of the staircase (padj_O $\quad 0.559, \mathrm{~d}_{\mathrm{O}} \quad 0.49$; $\mathrm{p}_{\text {Adj_ss }}<0.001, \mathrm{~d}_{\mathrm{SS}}$

1.41; Fig. 2), cadence was about 17 steps/min lower at steady state ( p $_{\text {Adj_O }} \quad 0.893, \mathrm{~d}_{\mathrm{O}} \quad 0.68$; $\mathrm{p}_{\text {Adj_ss }}<0.001, \mathrm{~d}_{\mathrm{SS}} \quad 1.59$; Fig. 2 ). No difference was found for step timing variability (padj_O $\quad 0.767, \mathrm{~d}_{\mathrm{O}} \quad 0.02$; p $\mathrm{p}_{\text {Adj_SS }} \quad 0.597, \mathrm{~d}_{\mathrm{SS}} \quad 0.54$ ) and acceleration RMS ( $\mathrm{p}_{\text {Adj_O }} \quad 0.597, \mathrm{~d}_{\mathrm{O}}$ 0.43).

When talking on the mobile phone, the pelvis (padj_o $0.038, \mathrm{~d}_{\mathrm{O}}$ 0.15 ; $\mathrm{p}_{\text {Adj_Ss }}<0.001, \mathrm{~d}_{\mathrm{SS}} \quad 1.20$; Fig. 3 ) and non-dominant foot jerk cost were lower ( p $_{\text {Adj_O }}<0.001, \mathrm{~d}_{\mathrm{O}} \quad 1.44$; $\mathrm{p}_{\text {Adj_ss }}<0.001$, $\mathrm{d}_{\mathrm{SS}} \quad 1.12$; Fig. 3). Inconsistent results between overall and steady-state were shown for the dominant foot: when using the phone, the jerk cost was higher overall and lower in steady state (p $\mathrm{p}_{\text {Adj_O }} \quad 0.043, \mathrm{~d}_{\mathrm{O}} \quad 0.21$; padj_ss $\quad 0.003$, d $\mathrm{d}_{\mathrm{SS}} \quad 1.14$; Fig. 3 ). The jerk cost was not different for the upper body (p $\mathrm{p}_{\text {Adj_O }} \quad 0.721, \mathrm{~d}_{\mathrm{O}} \quad 0.37$; $\mathrm{p}_{\text {Adj_SS }} \quad 0.893, \mathrm{~d}_{\mathrm{SS}} \quad 0.07$; Fig. 3).

No significant difference was shown for mean steady-state clearances in the lead or trail foot and in the vertical or horizontal direction ( $\mathrm{p}_{\text {Adj_Vertical_Lead }} \quad 0.578, \mathrm{~d}_{\text {Vertical_Lead }} \quad 0.43$; $\mathrm{p}_{\text {Adj_Vertical_Trail }} \quad 0.721$, $\mathrm{d}_{\text {Vertical_Trail }} \quad 0.27$; p pdj_Horizontal_Lead 0.597 , $\mathrm{d}_{\text {Horizontal_Lead }} \quad 0.29$; pAdj_Horizontal_Trail $\quad 0.597, \mathrm{~d}_{\text {Horizontal_Trail }} \quad 0.46$, Fig. 4).

Body velocity correlated with cadence $\left(\mathrm{r}_{\mathrm{O}} \quad 0.64, \mathrm{p}_{\mathrm{O}} \quad 0.044 ; \mathrm{r}_{\mathrm{SS}}\right.$ 0.61 pss 0.011$)$ and non-dominant foot jerk cost $\left(r_{O} \quad 0.66\right.$, $p_{O}$ 0.005 ; $\mathrm{r}_{\mathrm{SS}} \quad 0.63$, pss 0.008 ), but not with step timing variability ( $\mathrm{p}_{\mathrm{O}}$ 0.081 ; pss 0.093), acceleration RMS (pss 0.79), pelvis jerk cost ( $\mathrm{p}_{\mathrm{O}} \quad 0.91$; $\mathrm{p}_{\mathrm{SS}} \quad 0.14$ ), dominant foot jerk cost $\left(\mathrm{p}_{\mathrm{O}} \quad 0.064\right.$; $\mathrm{p}_{\mathrm{sS}}$ 0.069) or clearances ( $\mathrm{p}_{\text {Vertical_Lead }} \quad 0.17$; p pertical_Trail 0.27 ; $\mathrm{p}_{\text {Hor- }}$ izontal_Lead $\quad 0.37$; PHorizontal_Trail 0.48$)$.

\section{Discussion}

In this pilot study, a novel dual-task paradigm was used to test the effect of a cognitive task on motor performance during stair negotiation. We found that having a conversation over the phone induced changes in gait performance even in a group of young healthy participants.

\subsection{Talking on the phone induces measurable motor tuning}

With this dual task paradigm, the possible modifications in movement execution that the participants could implement were limited. It was not possible to greatly modify step length on the stairs or stop talking to prioritise movement control (Schaefer and Schumacher, 2010). In an in-person conversation, both individuals are aware of the difficulty of the dual task and may prioritise either task (Strayer and Johnston, 2001; Wickens et al., 2013). During a phone call, the participants were probably compelled to continue the conversation because they were not solely in control of the dialogue, as the operator was interacting with them remotely (Strayer and Johnston, 2001). The motor tuning measured included slower body velocity, lower cadence and jerk cost when talking on the mobile phone. These alterations are consistent with a decrease in locomotor performance (Hausdorff et al., 2001; Scott et al., 2015).

Reducing jerk cost is consistent with reducing the magnitude of changes in position, velocity and acceleration of movement. A lower jerk cost is consistent with a reduced amount of sensorimotor information required to control the movement (Hogan, 1984) and smoother locomotion (Hreljac, 1993). Moving more rapidly is usually associated with moving more smoothly in obstacle avoidance tasks (Hreljac, 1993). However, jerk cost and body velocity were both lower here when talking on a mobile phone. This result may appear counterintuitive and may reflect an underlying relationship between jerk cost and upper body velocity, which was not identified in the correlation analysis. However, it could also be speculated that this result reflects the priority of the neuromechanical system to simplify control of movement to cope with 

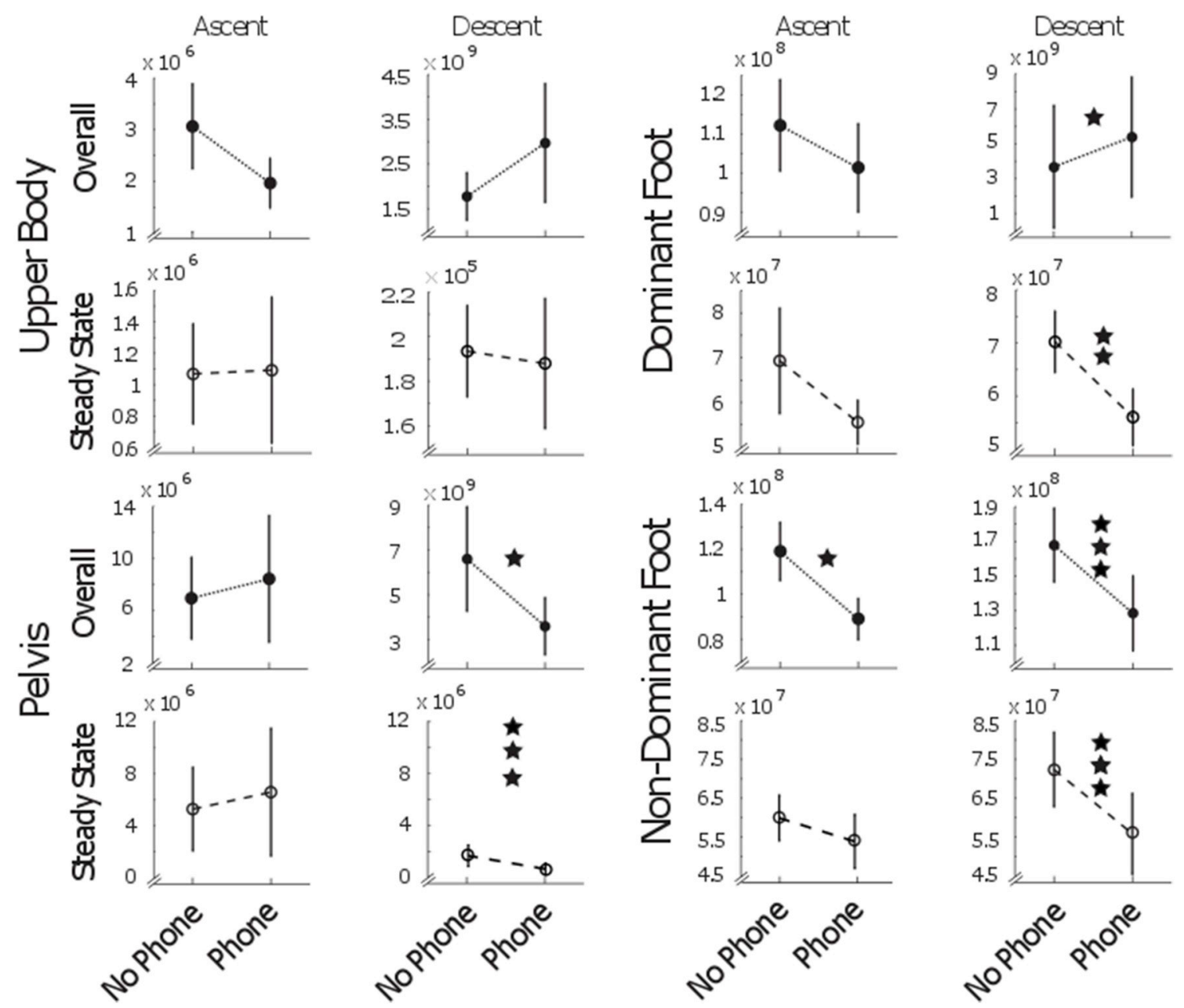

Fig. 3. Jerk cost.

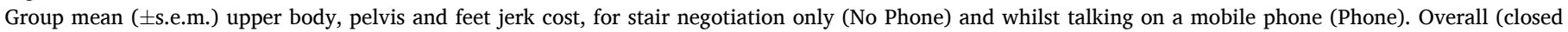
symbols, dotted lines) and steady state (open symbols, dashed) quantities are reported.

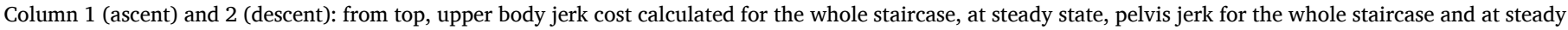
state.

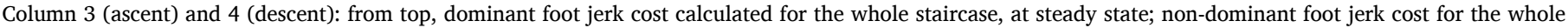
staircase and at steady state.

One star $\mathrm{p}<0.05$, two stars $\mathrm{p}<0.01$, three stars $\mathrm{p}<0.001$.

the increased demands, at the expense of slower movement. When the cost of movement control is reduced, the accuracy of the motor task may be compromised. This was confirmed by the higher step variability in ascent. In this experiment, the goal of the motor system was not fast or accurate execution of the task, but decreasing movement control difficulty.

\subsection{Why does motor performance deteriorate when talking on the phone?}

The current experiment is ecologically valid since it represents a common combination of tasks. The tasks chosen were unlikely to saturate information processing mechanisms (Wickens et al., 2013; Huang and Mercer, 2001) and were common daily activities for young participants. People often negotiate stairs whilst talking on the phone and cope with added cognitive and motor demands, such as other people in their path or carrying other objects. However, we measured alterations consistent with a decrease in locomotor performance.

Our results are consistent with previous work on texting/typing on a mobile and walking which showed impaired performance (Demura and Uchiyama, 2009; Licence et al., 2015; Ioannidou et al., 2017). Although in these studies texting and typing may introduce further difficulties for the participants due to the need for looking at the phone, we suggest that, as in our study, the deterioration in motor performance may be due to the overlap of the two tasks at different processing stages, which suggests a conflict at an information processing level. Previous dual-task paradigms using other cognitive tasks showed that attention and executive function are involved in walking (Lajoie et al., 1993; Coppin et al., 2006) and stair negotiation (Madehkhaksar and Egges, 2016; Telonio et al., 2014). Phone conversation and walking on stairs could conflict because they both need planning and error correction (Norman and Shallice, 2000). Whether this might be due, for example, to a bottleneck at the level of information processing (Allport et al., 1972; Pashler and Johnston, 1998) is difficult to determine at this point. 

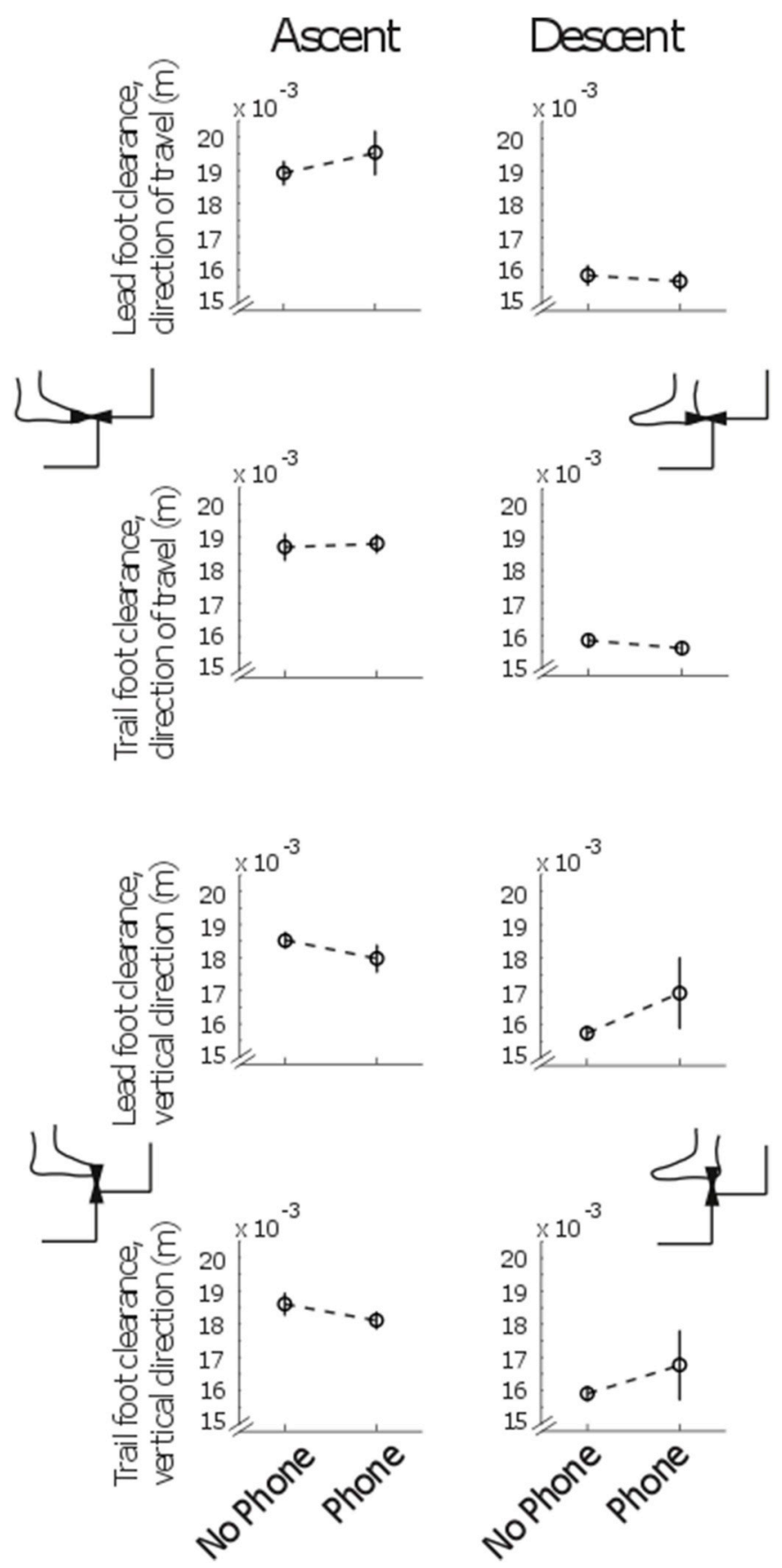

Fig. 4. Clearance.

Group mean ( s.e.m.) clearance for stair negotiation only (No Phone) and whilst talking on a mobile phone (Phone) in stair ascent (left) and descent (right). From top, lead foot clearance in direction of travel; trail foot clearance in direction of travel; lead foot clearance in vertical direction; trail foot clearance in vertical direction.

\subsection{Does motor tuning from talking on the phone increase stair fall risk?}

The impaired motor performance showed in this study, as evidenced by the lower body velocity with consequent increased exposure time and the increased movement variability in the dual task condition, are consistent with an increased risk of falling when talking on the phone while negotiating stairs (Hausdorff et al., 2001; Maki, 1997). The results showed here were indicative of adaptations in a young populations that reflect an increased demand of the task. Although we cannot establish a direct link between these adaptations and an increased fall risk in our participants, the changes measured here are consistent with typical locomotor adaptations showed by older and less intact groups, which were found to be consistent with an increased fall risk.

Gait variability is becoming an established measure of increased fall risk (Hausdorff et al., 2001; Decker et al., 2016) because, although individuals' gaits are different, differences between an individual's steps/strides may be indicative of a difficulty or of a compensatory mechanism, especially in older individuals or in populations with motor disorders. In the present study, young, healthy participants were tested and the increased gait variability when talking on the phone may be considered to indicate an increased fall risk for this population, in a similar way to that seen from studies in older adults. However, other quantities have more directly been linked with fall risk on stairs. For example, one main mechanism for a fall on stairs is tripping when the foot catches the edge of the step, especially during ascent (Templer, 1995). We did not observe significant differences in foot clearance between conditions. Nevertheless, it is surprising that in such a pilot study, young, healthy participants showed increased gait variability, which could be consistent with impaired stair performance.

Individual motor and cognitive capacity can also affect combined locomotor and cognitive adaptations. The present dual task would be more difficult if musculo-skeletal and/or cognitive abilities were decreased. This is a particularly important issue for an ageing population for whom both locomotor and cognitive abilities can slowly decline (Telonio et al., 2014) and individuals are less able to accurately judge their ability in relation to the task's demands. Older individuals could find a similar task more difficult than our healthy young adults. In addition, in a phone conversation, the interlocutor does not share the same environment as the person on the stairs, and thus the conversation is not adjusted or stopped to address the increasing locomotor needs, difficulty and risk. Further research is needed to understand the increased fall risk, the effect of talking on the phone and the underpinning mechanisms, especially in conditions more closely simulating real-life circumstances, such as when negotiating un-familiar, steep, narrow or poorly lit staircase. This field should aim to provide suggestions on accidents' and injuries' prevention, especially in individuals with reduced cognitive and/or physical capabilities.

\subsection{Study limitations}

This study presents several limitations. The order of conditions was not randomised because the experiment was part of a larger study. However, we believe that, because the staircase was of standard size, the participants' locomotor performance could be only mildly affected by familiarisation during the first trials and the randomisation was not crucial. In addition, this was a pilot study with a limited sample size, composed by young participants only. This test was designed as a feasibility study for further application in older people and possibly individuals with mild cognitive problems. Previous research shows that these groups may find stair negotiation more difficult cognitively (Ojha et al., 2009) and further work is needed to measure the performance using an ecological paradigm as the one used here.

The calculation of effect size has shown that, for the majority of the variables included in the analysis, the difference between conditions tested was between moderate and large. This indicates that large group size is not necessarily needed when investigating these tasks. In addition, conservative non parametric analyses were used to account for the reduced sample size. Thus, we believe that these encouraging results show that measurable changes in motor performance are seen even in young participants and justify future work in other populations.

In this study, we used a hand-held mobile phone because it is most common during walking. One may argue that the effective difficulty of the task (talk and walk) was increased by the act of holding the handset. However, we suggest that the differences measured are likely to be due to the added cognitive load of the phone conversation. In fact, previous experiments have shown that the peripheral factors, such as holding the 
handset, do not affect performance negatively (Nasar et al., 2008), suggesting that central factors are responsible for deterioration of performance (Ishigami and Klein, 2009). Furthermore, the demands of holding the handset are minimal for young healthy participants. As an example, even when children who just started walking (14 and 24-month-old) are measured whilst carrying an object in one hand, no difference was found in their main gait parameters, such as stride length, step length, step width, foot rotation and dynamic balance (Mangalindan et al., 2014).

It could be argued that the hand-held phone made handrail use more difficult. However, none of our young, healthy participants used the handrail or reported difficulties negotiating our experimental staircase, which was set to a dimension typical of public buildings and domestic dwellings (Government UK, 2013).

Step timing variability in this study was calculated for the seven step staircase. The number of steps included in this analysis might be considered relatively limited when compared to the number of steps needed to measure this quantity reliably in level walking (Almarwani et al., 2016; Konig et al., 2014). Although the minimum number of steps to reliably quantify this parameter has been established for level walking, it has not to our knowledge been established on stairs. Nevertheless, step timing variability was calculated and included here because it is relevant to evaluate gait performance and the potential relation with fall risk.

In conclusion, even young, healthy and cognitively intact participants modified their stair gait when talking on a mobile phone. Participants moved slowly, but more smoothly, reducing the motor control cost. This suggests that the priority was to simplify the control of the movement. Individuals with reduced cognitive and/or physical capabilities could find a similar task more difficult, and further work should quantify their potential fall risk in such situations.

\section{Author contributions}

The experiments were performed at the Laboratory of Biomechanics, School of Healthcare Science, Manchester Metropolitan University, Manchester, UK. All the authors contributed to design of the work. IDG contributed to the conception of the experiment, data acquisition, analysis and interpretation. IDG wrote the article. All authors contribute to the critical review of the manuscript and approved the final version.

\section{Funding}

This study was supported by the New Dynamics of Ageing (RES-35625-0037).

\section{Declaration of competing interest}

The authors declare that they have no known competing financial interests or personal relationships that could have appeared to influence the work reported in this paper.

\section{Acknowledgements}

We thank the anonymous participants for taking part to this experiment. We also thank Emily Kingdon for support with the data collection and Alexander Ireland for his technical support.

\section{References}

Allport, D.A., Antonis, B., Reynolds, P., 1972. On the division of attention: a disproof of the single channel hypothesis. Q. J. Exp. Psychol. 24, 225-235.

Almarwani, M., Perera, S., VanSwearingen, J.M., Sparto, P.J., Brach, J.S., 2016. The testretest reliability and minimal detectable change of spatial and temporal gait variability during usual over-ground walking for younger and older adults. Gait Posture 44, 94-99.
Coppin, A.K., Shumway-Cook, A., Saczynski, J.S., Patel, K.V., Ble, A., Ferrucci, L. Gurarlnik, J.M., 2006. Association of executive function and performance of dualtask physical tests among older adults: analyses from the InChianti study. Age Ageing 35, 619-624.

Decker, L.M., Cignetti, F., Hunt, N., Potter, J.F., Stergiou, N., Studenski, S.A., 2016 Effects of aging on the relationship between cognitive demand and step variability during dual-task walking. Age (Dordr) 38 (4), 363-375.

Demura, S., Uchiyama, M., 2009. Influence of cell phone email use on characteristics of gait. Eur. J. Sport Sci. 9 (5), 303-309, 2009.

Government UK, 2013. Protection from Falling, Collision and Impact Vol Approved Document K, Draft 2013 Edition. HM Government.

HASS and LASS Home \& Leisure Accident Surveillance System. Secion 3: HASS and LASS data. From. http://www.hassandlass.org.uk/reports/2002data.pdf.

Hatfield, J., Murphy, S., 2007. The effects of mobile phone use on pedestrian crossing behaviour at signalised and unsignalised intersections. Accid. Anal. Prev. 39, 197-205.

Hausdorff, J.M., Rios, D.A., Edelberg, H.K., 2001. Gait variability and fall risk in community-living older adults: a 1-year prospective study. Arch. Phys. Medicing. Rehabil. 82, 1050-1056.

Hogan, N., 1984. An organizing principle for a class of voluntary movements. J. Neurosci. 4, 2745-2754.

Hreljac, A., 1993. The relationship between smoothness and performance during the practice of a lower limb obstacle avoidance task. Biol. Cybern. 68, 375-379.

Huang, H.-J., Mercer, V., 2001. Dual-task methodology: applications in studies of cognitive and motor performance in adults and children. Pediatr. Phys. Ther. 13, 133-140.

IEEE, 1979. In: IEEE Acoustics, Speech, and Signal Processing Society. Digital Signal Processing Committee. Programs for Digital Signal Processing. IEEE Press, New York. Algorithm 5.1.

Ioannidou, F., Hermenes, F., Hodgson, T.L., 2017. Mind your step: the effects of mobile phone use on gaze behavior in stair climbing. J Technol Behav Sci 2, 109-120.

Ishigami, Y., Klein, R.M., 2009. Is a hands-free phone safer than a handheld phone? J. Saf. Res. 40 (2), 157-164.

Konig, N., Singh, N.B., Von Beckerath, J., Janke, L., Taylor, W.R., 2014. Is gait variability reliable? An assessment of spatio-temporal parameters of gait variability during continuous overground walking. Gait Posture 39 (1), 615-617.

Lajoie, Y., Teasdale, N., Bard, C., Fleury, M., 1993. Attentional demands for static and dynamic equilibrium. Exp. Brain Res. 97, 139-144.

Lamberg, E.M., Muratori, L.M., 2012. Cell phones change the way we walk. Gait Posture $35,688-690$.

Licence, S., Smith, R., McGuigan, M.P., Earnest, C.P., 2015. Gait pattern alterations during walking, texting, and walking and texting during cognitively distracting tasks while negotiating common pedestrian obstacles. PLoS One 10 (7), e0133281. https://doi.org/10.1371/journal.pone.0133281.

Madehkhaksar, F., Egges, A., 2016. Effect of dual task type on gait and dynamic stability during stair negotiation at different inclinations. Gait Posture 43, 114-119.

Maki, B.E., 1997. Gait Changes in older adults: predictors of falls or indicators of fear? J. Am. Geriatr. Soc. 45, 313-320.

Mangalindan, D.M.J., Schmuckler, M.A., Li, S.-A., 2014. The impact of object carriage on independent locomotion. Infant Behav. Dev. 37, 76-85.

Menz, H.B., Lord, S.R., Fitzpatrick, R.C., 2003. Acceleration patterns of the head and pelvis when walking on level and irregular surfaces. Gait Posture 18, 35-46.

Nasar, J., Hecht, P., Wener, R., 2008. Mobile telephones, distracted attention, and pedestrian safety. Accid. Anal. Prev. 40, 69-75.

Neider, M.B., McCarley, J.S., Crowell, J.A., Kaczmarski, H., Kramer, A.F., 2010. Pedestrians, vehicles, and cell phones. Accid. Anal. Prev. 42, 589-594.

Neider, M.B., Gaspar, J.G., McCarley, J.S., Crowell, J.A., Kaczmarski, H., Kramer, A.F., 2011. Walking and talking: dual-task effects on street crossing behaviour in older adults. Psychol. Aging 26, 260-268.

Nelson, W.L., 1983. Physical principles for economies of skilled movements. Biol. Cybern. 46, 135-147.

Norman, D.A., Shallice, T., 2000. Attention and action: willed and automatic control of behaviour (1980). In: Gazzaniga, M.S. (Ed.), Cognitive Neuroscience: a Reader. Blackwell, Oxford.

Ojha, H.A., Kern, R.W., Lin, C.-H.J., Winstein, C.J., 2009. Age affects the attentional demands of stair ambulation: evidence from a dual-task approach. Phys. Ther. 89 (10), 1080-1088.

Pashler, H., Johnston, J.C., 1998. Attentional limitations in dual-task perfromance. In: Pashler, H. (Ed.), Attention. Psychology Press/Erlbaum (UK) Taylor \& Francis, Hove, England.

Patten, C.J.D., Kircher, A., Ostlund, J., Nilsson, L., 2004. Using mobile telephones: cognitive workload and attention resources allocation. Accid. Anal. Prev. 36, 341-350.

Pellecchia, G.L., 2003. Postural sway increases with attentional demands of concurrent cognitive task. Gait Posture 18, 29-34.

Pijnappels, M., Bobbert, M.F., van Dieen, J.H., 2001. Changes in walking pattern caused by the possibility of a tripping reaction. Gait Posture 14, 11-18.

Plummer, P., Apple, S., Dowd, C., Keith, E., 2015. Texting and walking: effect of environmental setting and task prioritization on dual-task interference in healthy young adults. Gait Posture 41, 46-51.

Reeves, N.D., Spanjaard, M., Mohagheghi, A.A., Baltzopoulos, V., Maganaris, C.N., 2008. The demands of stair descent relative to maximum capacities in elderly and young adults. J. Electromyogr. Kinesiol. 18, 218-227.

Reeves, N.D., Spanjaard, M., Mohagheghi, A.A., Baltzopoulos, V., Maganaris, C.N., 2009. Older adults employ alternative strategies yo operate within their maximum capabilities when ascending stairs. J. Electromyogr. Kinesiol. 19, e57-e68. 
Riener, R., Rabuffetti, M., Frigo, C., 2002. Stair ascent and descent at different inclinations. Gait Posture 15, 32-44.

Schabrun, S.M., van den Hoorn, W., Moorcroft, A., Greenland, C., Hodges, P.W., 2014 Texting and walking: strategies for postural control and implications for safety. PLoS One 9 (1), e84312. https://doi.org/10.1371/journal.pone.0084312.

Schaefer, S., Schumacher, V., 2010. The interplay between cognitive and motor functioning in healthy older adults: findings from Dual-Task studies and suggestions for intervention. Gerontology 57, 239-246.

Schneider, K., Zernicke, R.F., 1989. Jerk-cost modulations during the practice of rapid arm movements. Biol. Cybern. 60, 221-230.

Schwebel, D.C., Stavrinos, D., Byington, K.W., Davis, T., O’Neal, E.E., de Jong, D., 2012 Distraction and pedestrian safety: how talking on the phone, texting, and listening to music impact crossing the street. Accid. Anal. Prev. 45, 266-271.

Scott, D., McLaughlin, P., Nicholson, G.C., Ebeling, P.R., Stuart, A.L., Kay, D., Sanders, K M., 2015. Changes in gait performance over several years are associated with recurrent fall status in community-dwelling older women at high risk of fracture. Age Ageing 44, 287-293.

Simoneau, G.G., Cavanagh, P.R., Ulbrecht, J.S., Leibowitz, H.W., Tyrrell, R.A., 1991. The influence of visual factors on fall-related kinematic variables during stair descent by older women. J. Gerontol. 46, 188-195.
Stavrinos, D., Byington, K.W., Schwebel, D.C., 2011. Distracted walking: cell phones increase injury risk for college pedestrian. J. Saf. Res. 42, 101-107.

Strayer, D.L., Johnston, W.A., 2001. Driven to distraction: dual-Task studies of simulated driving and conversing on a cellular telephone. Psychol. Sci. 12, 462-466.

Telonio, A., Blanchet, S., Maganaris, C.N., Baltzopoulos, V., Villeneuve, S., McFadyen, B. J., 2014. The division of visual attention affects the transition point from level walking to stair descent in healthy, active older adults. Exp. Gerontol. 20, 26-33.

Templer, J., 1995. The Staircase: Studies of Hazards, Falls, and Safer Design. MIT press.

Timmis, M.A., Bijl, H., Turner, K., Basevitch, I., Taylor, M.J.D., van Paridon, K.N., 2017. The impact of mobile phone use on where we look and how we walk when negotiating floor based obstacles. PLoS One 12 (6), e0179802. https://doi.org/ 10.1371/journal.pone.0179802.

Verghese, J., Kuslansky, G., Holtzer, R., Katz, M., Xue, X., Buschke, H., Pahor, M., 2007. Walking while talking: effect of task prioritization in the elderly. Arch. Phys. Med. Rehabil. 88, 50-53.

Wickens, C.D., Hollands, J.G., Banbury, S., Parasuraman, R., 2013. Engineering Psychology and Human Performance, fourth ed. edn. Pearson Education, Inc, New Jersey.

Woollacott, M., Shumway-Cook, A., 2002. Attention and the control of posture and gait: a review of an emerging area of research. Gait Posture 16, 1-14. 